\title{
A Multi-Model Approach for Simulation-Based Digital Twin in Resilient Services
}

\author{
ARNIS LEKTAUERS \\ Department of Modelling and \\ Simulation \\ Riga Technical University \\ Kalku Street 1, Riga \\ LATVIA
}

\author{
ANDREJS ROMANOVS \\ Department of Modelling and \\ Simulation \\ Riga Technical University \\ Kalku Street 1, Riga \\ LATVIA
}

\author{
JELENA PECERSKA \\ Department of Modelling and \\ Simulation \\ Riga Technical University \\ Kalku Street 1, Riga \\ LATVIA
}

\author{
JANIS GRABIS \\ Department of Management \\ Information Technology \\ Riga Technical University \\ Kalku Street 1, Riga \\ LATVIA
}

\author{
VITALIJS BOLSAKOVS \\ Department of Modelling and \\ Simulation \\ Riga Technical University \\ Kalku Street 1, Riga \\ LATVIA
}

\author{
ARTIS TEILANS \\ Rezekne Academy of \\ Technologies \\ Atbrīvošanas aleja 115, \\ Rezekne, \\ LATVIA
}

\begin{abstract}
Complex cyber-physical systems demand integrated solution approaches. The current work presents a multi-model approach for simulation-based digital twins as a formal and technological foundation for the analysis and improvement of resilient services. The given approach has several significant benefits including the possibility to conduct interactive simulations and experiments based on systems engineering principles, to share data across multiple data sources and storages, to manage operations in real-time, as well as to enable collaboration between the users in an integrated web platform. The proposal is illustrated on the use cases of secure telemedicine services and secure remote workplace.
\end{abstract}

Key-Words: - Multi-Modelling, Simulation, Digital Twin, Resilient Services

Received: August 12, 2020. Revised: January 3, 2021. Accepted: January 19, 2021. Published: February 20, 2021.

\section{Introduction}

The digital transformation is a critical success factor in today's rapidly developing and changing economic, industrial, and social environment. Digital twins there are increasingly important components improving the ability to manage complex cyber-physical systems more efficiently [1].

The concept of digital twin is widely used today, in the same time the basic foundation for digital twin is remaining relatively stable since its inception in 2002 when the idea of digital twin was presented for the first time at the University of Michigan for the formation of a Product Lifecycle Management (PLM) centre [2].

Cyber-physical systems consist of multiple spatially and temporally interrelated and interdependent subsystems, components, and related services. To provide and support a resilience of these subsystems, a holistic system approach is necessary by involving advanced decision-making tools. And therefore, complex cyber-physical systems demand integrated solution approaches.

Modelling and Simulation (M\&S) for a long time is used as a decision-making tool for a wide range of complex problem solutions including the application in digital twins. However, due to the overall multidimensional nature of real-world complex systems traditional single type M\&S methodologies, e.g., Discrete Event Simulation (DES) [3], System Dynamics (SD) [4], Agent-Based Simulation (ABS) methodologies can face serious challenges to correctly represent such systems at different abstraction, temporal or spatial levels. To overcome such issues, a multitude of hybrid or multi-model M\&S approaches have been invented for modelling and simulation of different facets of complex systems.

The objective of the proposed multi-model approach is to provide a digital twin supported technological foundation for the analysis and improvement of resilient services. The concept, as well as the 
experimental implementation are developed within the project "Advanced Resilience Technologies for Secure Services" (ARTSS) at the Riga Technical University, Latvia [5]. The proposal is illustrated on the use cases of secure telemedicine services and secure remote workplace.

This paper is organized as follows. Section 2 presents the state of the art in digital twins. Section 3 presents the multi-model approach for simulation-based digital twin. Section 4 presents the designing of digital twin for resilient services. Section 5 offers concluding comments on the benefits and future prospects of simulation-based digital twins.

\section{State of the Art in Dgital Twins}

\subsection{The Concept of Digital Twin}

Although the term "digital twin" has not been formally defined in international standards, there are many definitions, ranging from abstract and vague ones such as "a set of virtual information constructs that fully describes a potential or actual physical manufactured product from the micro atomic level to the macro geometrical level" [2] or "digital representation, sufficient to meet the requirements of a set of use cases" [6], and up to solutionoriented, e.g., "digital representation of physical individuals as well as of virtual entities in an information framework that interconnects traditionally separated elements and provides an integrated view throughout life cycles" [7]. A very close approach to digital twin is model-based commissioning for automated control system design [8].

In frame of the current research the following digital twin definition is accepted by the research team - "a dynamic virtual representation of a physical object or system across its lifecycle, using real-time data to enable understanding, learning and reasoning" [9]. This definition is obviously associated with the conventional definition of simulation model [3]. A model is defined as a representation of a system for the purpose of studying that system. Only some aspects of the system are represented in the model and the model provides information about the system. The main component that distinguishes a digital twin from any other digital model is its connection to the physical twin [10] that is represented by digital twin and simulation model. Both use the data from the real system and provide information. Digital twin changes over time being adjusted to the phase of the physical twin's lifecycle [11]. The simulation model usually is tailored to solve a particular problem, thus there may be different simulation models of the same system.

There are three important parts in the digital twin of an object [1], [12]:

- a data-driven executable virtual model of the object,

- an evolving set of data relating to the object, and

- means of dynamically updating or adjusting the model in accordance with the data.

More recent research outlines more important parts, but it is obvious that both definition and requirements are not defined very strictly.

The scope of the digital twin depends on at what stage of the product life cycle the simulation takes place. Siemens distinguishes three types of digital twins: Product, Production, and Performance; the combination and integration of the three digital twins as they evolve together is known as the digital thread [13]:

- Product digital twins provide a virtual-physical connection showing how a product performs under various conditions and make adjustments in the virtual world to ensure that the physical product will perform exactly as planned.

- Production digital twins can validate how well a manufacturing process will work on the shop floor before starting the production; production can be optimized by digital twins of all manufacturing equipment, to prevent costly downtime, and to predict the necessity of preventative maintenance.

- Performance digital twins captures the massive amounts of raw data regarding products and factories utilization and analyses it in order to create new business opportunities, gain insight to improve virtual models, improve product and production system efficiency.

Gartner expects one-third of midsize and large IoT companies to have at least one digital twin by 2023 . According to a recent industrial survey [14], 31\% of respondents use digital twins to enhance the safety of their employees or customers, $27 \%$ of companies plan to use digital twins as autonomous equipment, robots or vehicles. Such a fast pace of implementation of digital twins is explained both by the value of the technology itself for the business, and by active marketing and training programs of vendors.

\subsection{Digital Twin Usage Examples}

Digital twins have found their way into a wide variety of areas. One of the most important functions is to improve production processes, identify bottlenecks (system components, processes, and other assets), test prospective solutions, 
simulate the results of interactions between components, and predict stochastic changes that can occur through various components performing operations. This simulation saves organizations resources and funds to test working hypotheses in practice. The perspectives of digital twins application [10] in various areas can be assumed in three dimensions: (A) potential futures with strategy and planning support, 'What if?' scenarios, predictive and preventive maintenance. (B) current state with intervention, real-time status monitoring, control, and prognostics to optimize performance and safety of assets, and (C) history record-keeping and learning from the past.

Not only temporal and scale aspects can be included into the areas benefiting from the implementation of digital twins, various human activity areas, such as manufacturing, supply chain management, healthcare etc. can be added too [13], [15]-[17].

Almost supporting the whole products life cycle, the applications of digital twins in manufacturing start with product design, when companies use digital twins to design different products options, making it more effective to deliver personalized products and services to their customers. During the product manufacturing, digital twins provide an opportunity to evaluate and test the feasibility of developed products before launch, which results in either start production or switching to more feasible products. Finally, for shop floor performance improvement and predictive maintenance the digital twin can be used to monitor and analyse end-products and check which products are defected or have lower performance than intended.

Digital twins are also widely used in supply chains and logistics, helping to create logistics networks through the digital twin of the road network, carrying information about traffic conditions, road layout and construction; helping to optimize warehouse design and performance, enabling companies to select the most efficient warehouse design for maximum productivity; and improve product delivery security by virtualizing and analysing various packaging conditions and packaging material characteristics.

In the healthcare area digital twins can support providers to virtualize and optimize patient care, cost, and productivity. The main usages of twins are: (A) improving the operational efficiency of healthcare activities through the creation of a digital twin of the hospital, operational strategies, capabilities, staffing and service modes; and (B) improving personal patient care services to model the physiological or behavioural characteristics of patients, genome code, and the like so that healthcare companies can provide personalized care and unique medicines for each patient.

Some recent research results are overviewed further in this paper.

\subsection{Modelling Approaches and Tools for Modelling of Digital Twin}

A variety of approaches to modelling is supposed in most research projects. Digital twins may use different approaches to modelling, including [10]:

- Geometric and geospatial modelling.

- Computational/mathematical/numerical modelling.

- Artificial intelligence and machine learning; 'learning systems'.

The above-mentioned cases provide the simulation information as well. The supply chain case authors used an actor language, where each actor has a behaviour that can be modelled as a state machine [18]. The same approach is used by the authors in the University case [19].

The researchers are not limited with a specific simulation paradigm, technique, or software. Some software decisions are considered in the next section.

\subsubsection{Tools for Modelling of Digital Twin}

Within scientific literature application of digital twins is reviewed in various fields of industry, economics, and medicine. However, most of the scientific literature related to use of digital twin very rarely devotes its attention to the details of development of models for digital twin. Often only the concept of digital twin is described without details of the model to simulate an original system.

Requirements for simulation in digital twin are specific and justified by the scope of modelling within the concept of digital twin [11]. Models for digital twin usually are required to simulate the original system as accurately as possible. Moreover, models are required to be easily and relatively quickly changeable to follow changes and improvements in the original system, so that work and experiments with the digital twin would be the same as experiments with the original system. Also, one of requirements for modelling of digital twin is integration of models in the whole lifecycle of the digital twin, providing a convenient interface for controlling and impacting the model nearly the same as the original system.

Thus, traditional bottom-up approach in development of the simulation model could be struggled here due to model update cycle could be time-consuming here. For digital twin in particular 
scopes even application of data-driven models is proposed as these can be quickly adaptable to follow the changes of the replicated system, although usually providing only black box models [12].

Moreover, it is mentioned that development of the digital twin could include composition of digital twins of subsystems of replicated system, which relates to decomposition approach in modelling.

The reviews given in the [20] and [21] provide wide research on the technologies and tools in simulation and modelling of digital twins. Respectively, research in [21] provides following tools as applicable for modelling of digital twin (with notice to the behaviour modelling, as the most actual within current research): ANSYS Twin Builder; Dymola System Engineering; ADAMS (more concentrating on dynamics of mechanics); Simuworks (concentrating on hydrodynamics); MATLAB Simulink; MWorks (concentrates on neurophysiology); OpenModelica; SimulationX (concentrates on technical systems); other tools (mostly for simulation of industrial and technical systems).

Within this research an implementation of the simulation models realized in the Python environment are proposed. Part of the models will be directly implemented as Python programs; part will be automatically generated by modelling computer-aided tools. Such implementation will provide an easy cross-platform integration for the multi-modelling tool.

\section{Multi-Model Approach}

As already was stated in the Introduction, it can be difficult to model at an appropriate level of abstraction or resolution using a single $M \& S$ method. As most real-world cases are complex, it is more convenient to model different parts of a system with different methods by combining the used methods in one hybrid composite model. For example, depending on the detail of level of the available input data secure network services or secure remote workplace services efficiently can be modelled by using agent-based approach. But if the available input data is aggregated at higher level, then a macro level simulation approach, e.g., system dynamics can be more appropriate there.

\subsection{Agent-based Simulation}

Agent-based simulation approach is considered as a suitable approach for the development of digital twin of particular use cases in this research. Agentbased simulation paradigm implies simulation of many agents which are modelled simultaneously, and which continuously interact between themselves, and with external environment. Each agent has a relatively simple behaviour, but more interesting is the whole behaviour of the system which could not be inducted from a single agent's behaviour but can be obtained in simulation.

Within this research application of multi-agent approach is considered appropriate for development of digital twins of secure foundational services and secure remote workplace. In both cases a number of separate agents with well-known behaviour and structure could be distinguished in a system (e.g. employees, which have to work in the remote environment), but the whole system itself is designed as a complex structure based on the interaction of the agents (e.g. communication network of these agents). Moreover, the multi-agent approach here allows a bottom-up design of digital twin, where behaviour of low-level elements of the system is well known, but high-level behaviour patterns could be hard to distinguish and define in the model directly.

\subsection{System Dynamics}

System dynamics (SD) [4] is considered as another suitable approach for the development of digital twins. System dynamics models focus on the system processes at the high abstraction level, not concentrate on the details. The study of system processes by system dynamics models is most often used to solve strategic tasks.

The basic SD model is a system of equations that includes two main types of equations - the level and flow equations. The generic model elements are levels and flows, and the details of the model are provided by auxiliary elements - variables and information links. The levels can change only from flows. The solutions to the system of equations after a fixed time step provide the new model state. The most powerful feature of SD simulations is information feedback.

The system of equations corresponds to the structure of the system and reflects the cause-effect relationship in a relatively simple mathematical form. The system of equations ensures a continuous representation of the interaction of the model elements. The equation system comes together with certain rules that determine the sequence of solving these equations. The system dynamics models are the discrete time system simulators.

The simulation model in most SD modelling environments is available in a form of level and flow diagram with auxiliary variables and information links between the model elements. The diagrams visualise the processes in clear form. 
In most cases, both system dynamics and discrete event approaches can be used to simulate processes. For the purposes of this research it was not necessary to observe individual flow objects, thus the choice of the approach between discrete-event and SD was affected by the claim of SD software developer about the model integration possibility into end-user web applications [22].

\subsection{Technology Integration}

In this work we propose a hybrid multi-model concept for simulation-based digital twin of resilient services, shown in Fig. 1.

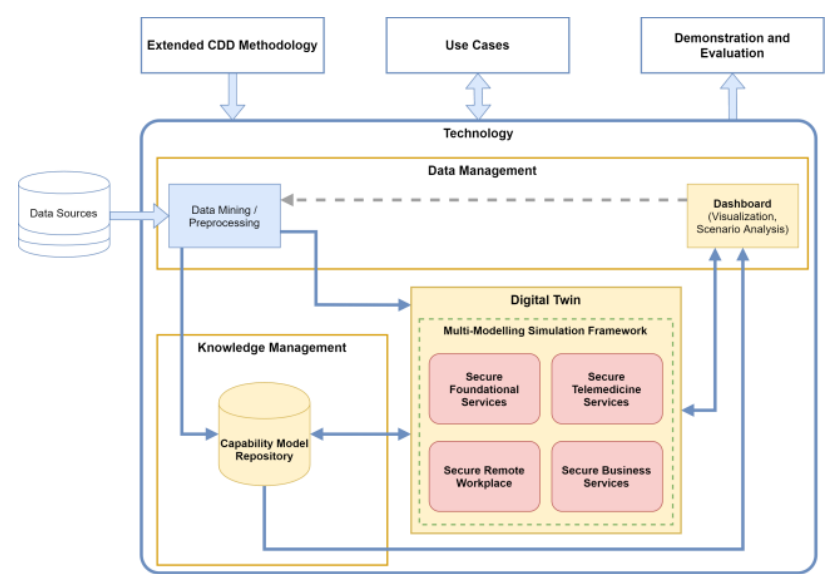

Fig. 1. Concept of simulation-based digital twin technology

The methodology of the technology integration is based on an extension of the Capability Driven Development (CDD) method [23]. The CDD method supports the development, delivery and management of organisation and information system capabilities. The extended method preserves the main principles and elements of the CDD methodology and supplements these with several new elements and method components:

- Ecosystem perspective;

- Digital twin support;

- Security and resilience goals and knowledge sharing;

- Learning support.

The multi-modelling simulation framework provides a foundation for a digital twin acting as a digital replica of the real-world processes to be analysed and controlled. The digital twin is gathering the input data from the data management and knowledge management modules. The purpose of the data management module is to support an automatic input data mining and pre-processing, as well as to represent the output results generated by the digital twin to the end-user in a dashboard. The knowledge management module stores CDD models used for automatic configuration and adaptation of the digital twin according to specific goals, capabilities, and adjustments.

The digital twin module is a composite multimodelling simulation framework implementing a generic concept of modular composite digital twin [6] consisting of multiple discrete digital twins that are representing the lowest level of abstraction still sufficient to meet the requirements of a specific use case. A composite digital twin can be formally specified based on the principles of the system theory [24] as follows:

$$
D T_{C}=\left\langle T, X, \Omega, Y, D, D T_{D}\right\rangle,
$$

where $T$ is a time base, $X$ is a set of input values, $\Omega$ is a set of states, $Y$ is a set of output values, $D$ is a set of discrete digital twin component references, $D T_{D}=\left\{D T_{d} \mid d \in D\right\}$ is a set of discrete digital twin components.

The digital twin module represents four use cases covered by the given research study within the ARTSS project [5] with corresponding discrete digital twins in an integrated technological platform:

- Secure foundational services,

- Secure telemedicine services,

- Secure remote workplace,

- Secure business services.

Each discrete digital twin $D T_{d}$ is an adaptive autonomous subsystem of the composite digital twin $D T_{c}$ that can be formally expressed in a form of Difference Algebraic Equations (DAEs) [25]:

$$
\begin{aligned}
& F\left(x^{+}(t), x(t), u(t), w(t), \pi(x(t)), t, \theta\right)=0, \\
& y(t)=h(x(t), v(t), \theta),
\end{aligned}
$$

where $F$ is a vector valued function, $x^{+}(t) \in \Omega$ is the state of the system at the next time instance, $x(t) \in \Omega$ is a vector containing the internal state variables, $u(t) \in X$ is a vector of control input variables, $w(t)$ is a vector of disturbance realizations e.g. faults, $\pi(x(t))$ is autonomous control function, $t \in T, t_{0} \leq t<H$ is time index within the planning horizon $H, \theta$ is a vector of model parameters, $y(t) \in Y$ is a vector of measured variables, $h(x(t), v(t), \theta)$ is sensing function measuring the output of the system, $v(t)$ is a vector of sensing disturbances to express e.g. sensor noise or package drop in the communication network. 


\section{Designing Digital Twin for Resilient Services}

The digital twin implementation (Fig. 2.) is focused on the support of multi-modelling approach for various use cases requiring different simulation methods. An important aspect there is also the support of asynchronous distributed task processing allowing to efficiently coordinate different discrete digital twin components in a distributed cloud environment. The architecture is based on freely available high-quality open source software tools to make the implementation efficiently extendable, maintainable, and available for integration with external systems.

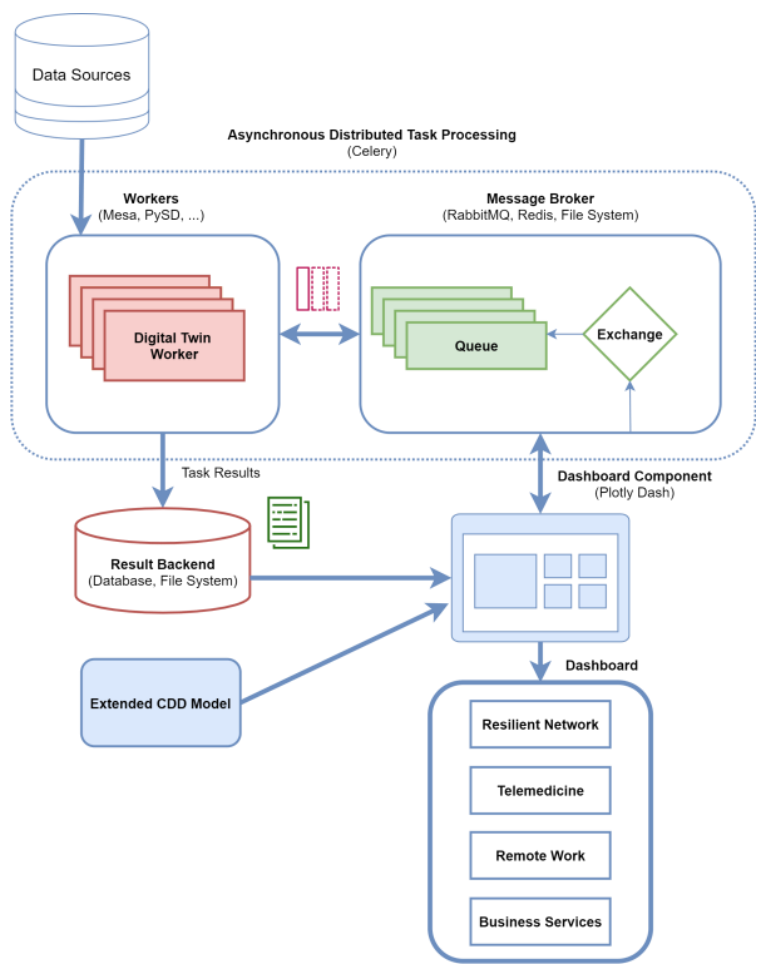

Fig. 2. Architecture of the digital twin implementation

The dashboard component is the front-end for the digital twin module implementing the concept of Capability Navigation Application (CNA) as a part of the extended CDD methodology [23].

Although the design and implementation provides an experimental support for four resilient service use cases, due to the article size limit there are described two use cases in the next subsections - the digital twins of secure telemedicine services and secure remote workplace.

\subsection{Secure Telemedicine Services}

The goal of this section is to introduce the digital twin for the secure telemedicine services based on the extended Capability Driven Development (CDD) methodology. The tasks of this section are to create the concept of the digital twin for the secure telemedicine services; to introduce the simulation framework; to verify and validate the digital twin and to provide the insight into results interpretation.

\subsubsection{The concept of the digital twin for the secure telemedicine services}

The concept of the digital twin for the secure telemedicine services is formulated in the terms of capabilities that enable an enterprise to achieve a business goal in a certain context [23]. The enterprise under consideration is a practice of the general practitioner (PGP). The business goal is the secure and stable telemedicine service both under normal and emergency conditions. The concept is based upon the informal capability model, created in the ARTSS project [5].

The practice of the general practitioner (PGP) as an enterprise operates in the strict accordance with the law and regulations by the Latvian Cabinet of Ministers. Therefore, the arbitrary interference in this activity is unacceptable. The PGP must provide the access to the primary medical services for all the registered patients in full, and the waiting time should not exceed 5 working days [26].

The question of the efficiency of using the main resource of the PGP - working time - is not analysed from the point of view of sufficiency or insufficiency. The subject of the research is the efficient allocation of the main resource depending on the demand and the context. The main problem is the elimination of the insufficient capability impact on the performance. The parameters of the DT are the estimates of the capabilities of providing the particular services and the patient flow historical data. The main performance indicator is the unsatisfied demand for the remote service; the additional indicator is the required working time.

The business process under consideration is the patient's process in the PGP. The main types of services in the PGP are on-site consultations and remote consultations provided by general practitioner, and the medical assistant consultations. If the remote consultation is not possible, it is redirected to the on-site consultations. The simplified patient's process description can be formalised in the form of activity diagram as shown in Fig. 3. The activity diagram expands if there are new activities in the process. The conditional activities are executed only if there is a sufficient capability for providing these activities. The capability to provide the remote consultation is evaluated using user's opinion data on various 
particular capabilities. The user, who has information about the process and the activities in the PGP, can perform the simple dashboard-based data input.

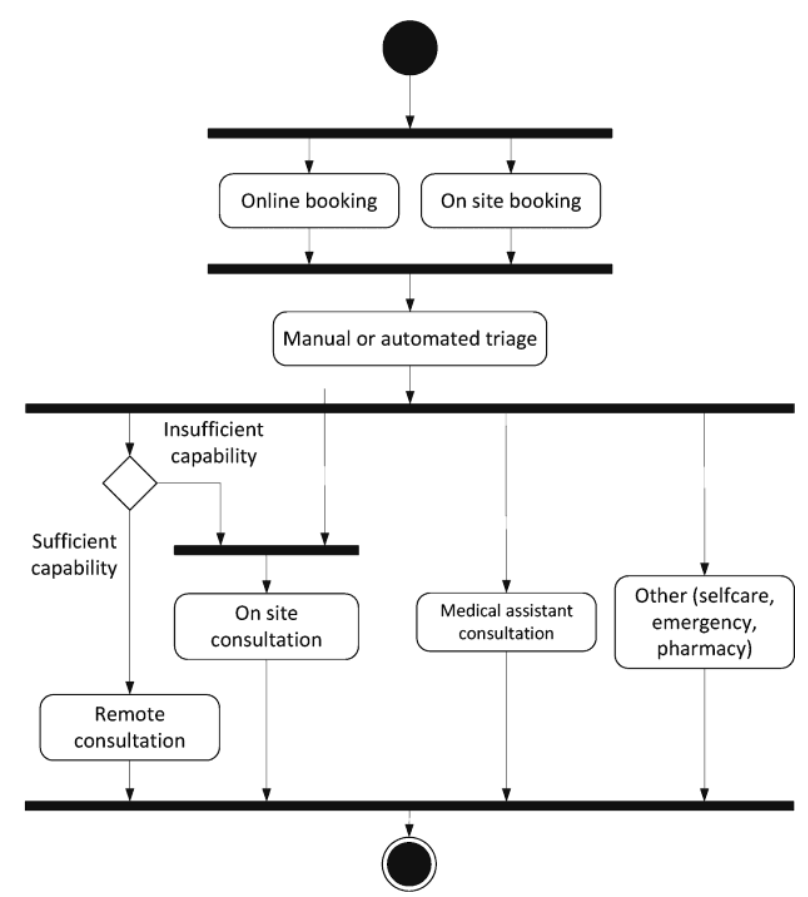

Fig. 3. The patient's process activity diagram

We note the inexpediency of creating a real-time synchronous DT. To assess the necessary resource volume, a concept is formulated at the high level of abstraction. This approach enables the assessment of the effectiveness of resource allocation, as well as the detection of the lack of capabilities in the context of providing the necessary services in the long term.

The conceptual model of the PGP involves the information about the problem, goals of DT building, input data - patient flow and user's opinion, the structure of the process, capability model, output performance indicators, as well as the simplifications and assumptions made for the model creation.

The activity diagram shows that the implementation of the DT simulator is possible in such simulation paradigms as discrete-event system simulation and discrete time system simulation. Since there is no need to analyse the process of each individual patient, the project adopted the discrete time simulation paradigm for implementation of the DT simulator.

The capability concept is interpreted as a modified Bayesian belief network (BBN). BBN modification based on the estimates of capabilities using the likelihood rating scale is used. The user's opinion is taken into account in the form of capability ratings.
The user's opinion is included into the calculation of the general practice capability to provide the required service

The digital twin incorporates the capability BBN, patient flow simulator, user's opinion on capabilities and key indicators to evaluate the performance. The concept of the DT incorporating the system dynamics (SD) patient's process simulator is shown in Fig. 4.

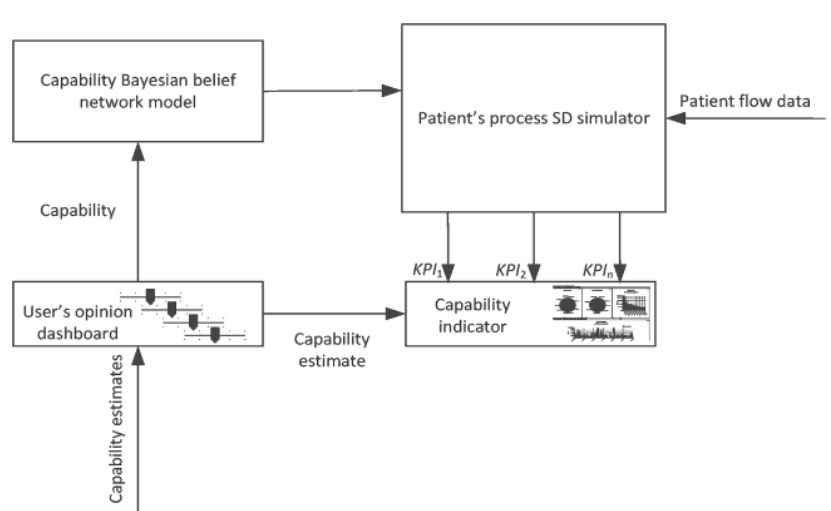

Fig. 4. The telemedicine service digital twin concept

\subsubsection{The patient's process simulator for the practice of general practitioner}

General information and data about the annual patient flow in the practice of the general practitioner are obtained from the publications of Ministry of Health of the Republic of Latvia and from the official data portals [26]-[28] and are shown in Table 1.

Table 1. General information about patient flow at the general practitioner

\begin{tabular}{|l|r|}
\hline Parameter & Value \\
\hline $\begin{array}{l}\text { Average number of registered patients per } \\
1 \text { general practitioner }\end{array}$ & 1564 \\
\hline Average number of visits per year & 3.1 \\
\hline Average number of working days per year & 232 \\
\hline Average consultation duration (min) & 15 \\
\hline Number of PGP in Latvia & 1206 \\
\hline
\end{tabular}

These values are the basic parameters of the simulator. Particular general practice parameters as an input from the dashboard are applicable as well. In the context of the COVID-19 pandemic, the information is necessary about the emergency patient flow who need only remote service. We do not consider cases where patients are hospitalized. 
The increased workload of the PGP can be roughly estimated based on the data on the number of cases and the number of PGP in the country. It is assumed that the average duration of the disease is 20 days, during this time the patient on average remotely consults with a general practitioner 5 times. The data, which is available from February 29 to December 10, 2020 is forecasted via polynomial curve extrapolation up to February 28, 2021. The data is used to simulate the emergency patient flow (Fig. 5).

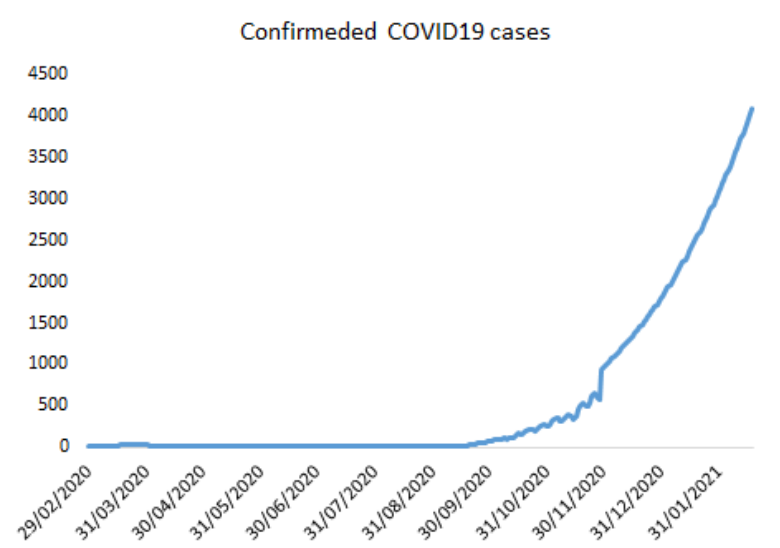

Fig. 5. Confirmed COVID19 cases in Latvia with the extrapolation from the 10th of December 2020

\subsubsection{The patient flow simulator for the practice of general practitioner}

The system dynamics simulator is verified by using calculated capability values and is validated by using a particular user's opinion input. The fragment of the SD simulator with the user's opinion dashboard is provided in Fig. 6 .
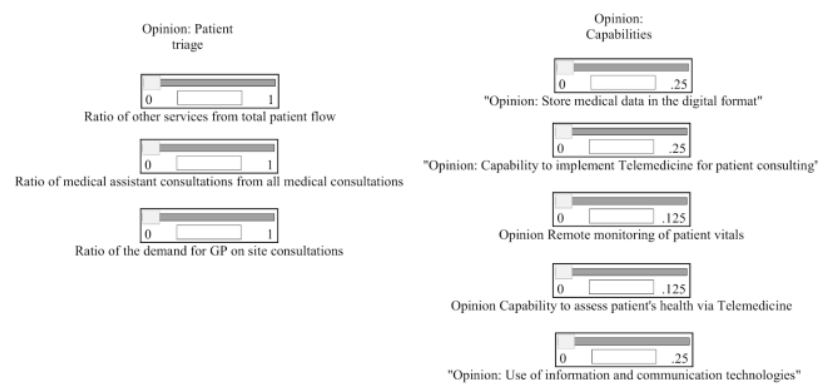

Fig. 6. The fragment of the SD simulator with opinion dashboard

\subsubsection{The simulation results analysis and future work}

The simulations are performed under conditions of the insufficient capability, based on the user's opinion. The estimate of the unsatisfied remote demand result is obtained in range from 114 to 160 cases per year. The histogram of the obtained estimate for the indicator based on 200 runs is provided in Fig. 7.

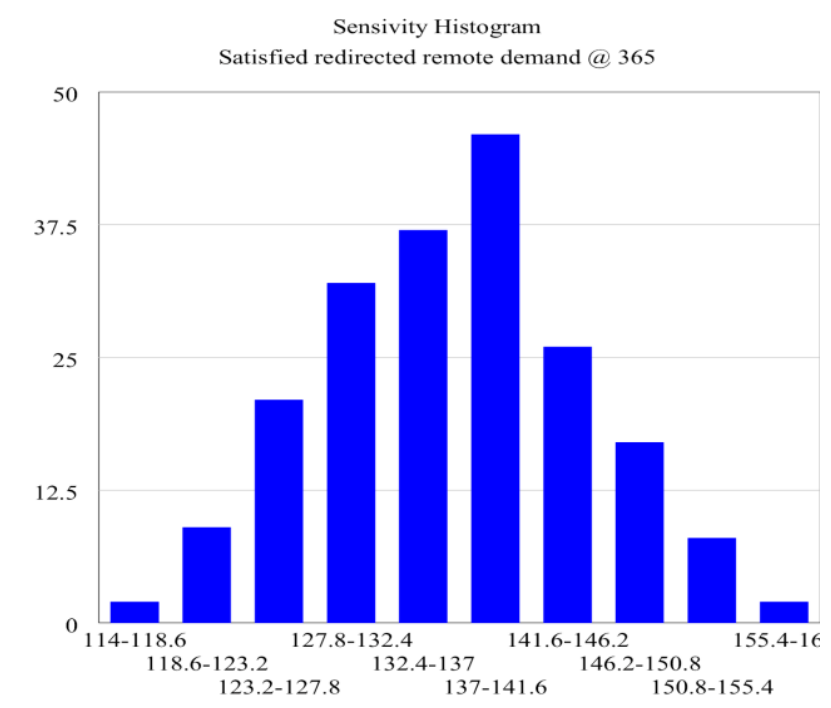

Fig. 7. The resulting histogram of the volume of unsatisfied remote demand

The demand for video/audio consultation is not satisfied in full in average for the capability level 0.8 . Unsatisfied demand for remote consultation is approximately $13 \%$ from the total demand for remote consultations. This result shows that the capability impact on the performance is significant and the resource allocation for remote consultation is not sufficient.

This simulator deals with the resource usage and is not analysing the consultation activity of the general practitioner. However, the capability to provide the remote service is not only the combination of technical capacity and ability. This capability depends on the workload pressure on the general practitioner as well. It is possible to use the simulator in the traditional way in order to identify the feedback of workload impact on the performance of the general practitioner.

The possibility to analyse the service time increase is analysed taking into account the Perceived Stress Scale or Yerkes-Dodson empirical law that describes the relation between workload and performance [29]. The workload pressure is introduced with relation to the average service rate as a graphical function of the performance against the workload. The complexity and familiarity of the task are not taken into account. The evaluation of the performance expectation is in Fig. 8, where the performance is evaluated after 200 simulation runs. The dark blue area gives the 95\% confidence interval of performance expectation. This simulator 
estimates the necessity of extra resources at average level of $13 \%$ while the emergency is in progress.

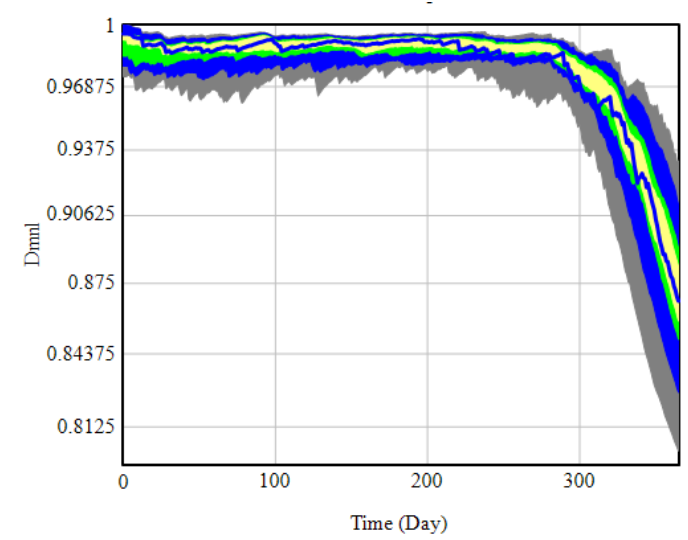

Fig.8. The performance expectation time series using the logarithmic scale y-axis

The digital twin concept is realised based on the ARTSS project methodology. The asynchronous digital twin uses the accumulated real-time information for monitoring the quality of service delivery. The digital twin uses the system dynamics simulator for both monitoring the quality of service delivery, for the capability impact evaluation on performance and workload pressure impact on performance of the practice of general practitioner.

\subsection{Secure Remote Workplace}

This section describes development and experiments with the secure remote workplace digital twin. Secure remote workplace environment is modelled here as a system, where a number of employees connected by communication tools and channels can exchange messages and information between each other, thus producing the complex behaviour from aspects of remote workplace on the complex behaviour of the whole enterprise as a system.

\subsubsection{Conceptual Model}

To design a digital twin of secure remote workplace following classes of agents of the modelled system are distinguished in conceptual model:

- Employee - performs all behaviour in the context of communication of organisation's or enterprise's individual worker.

- Message - communication event between two Employee agents.

- Infrastructure - infrastructure related agents, such as networks, servers, etc.

The following general conceptual model of the simulated system is proposed as given in Fig. 9.

Different context could be applied to employees, regarding if they are working remotely or from the office. Communication links between employee agents dynamically change in the simulation time, number of agents and proportion of remote agents could be different.

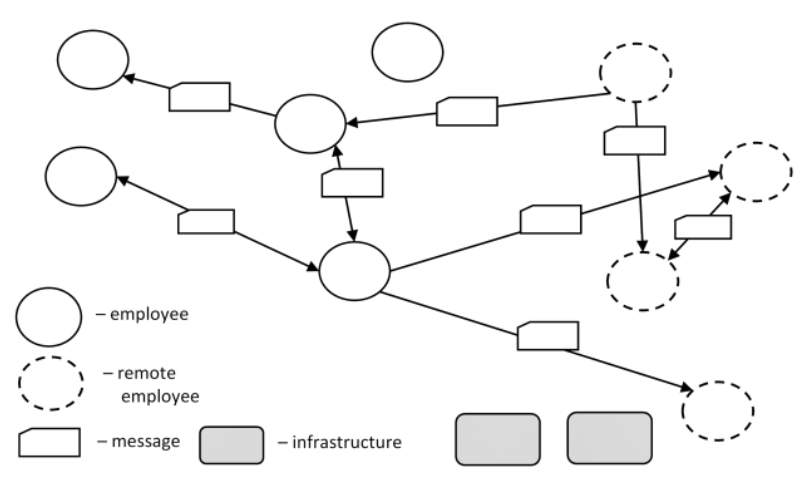

Fig. 9. Conceptual model of communications in the context of remote workplace

The digital twin is interpreted here as given in (4):

$$
R W=\langle E M, M E, I N\rangle,
$$

where $R W$ is digital twin for remote workplace, $E M$ - is a set of employees, $M E$ - a set of messages and $I N-$ a set of infrastructure objects. The sets $E M$ and $M E$ itself could be defined as given in (5) and (6):

$$
\begin{aligned}
& E M=\left\{e m_{1}, e m_{2}, \ldots, e m_{n}\right\}, \\
& M E=\left\{m e_{1}, m e_{2}, \ldots, m e_{m}\right\},
\end{aligned}
$$

where $e m_{\mathrm{i}}$ is a particular $i$-th employee, $n$ is a total number of employees, $m e_{j}$ particular $j$-th message and $m$ a total number of messages.

The main element of the proposed agent-based model for remote workplace digital twin is Employee agent, which implies a behaviour, where all Employee agents are periodically communicating with other agents using different communication channels and communication types. Each employee $\mathrm{em}$ from (5) itself if formalised as given in (7):

$$
e m=\langle r w, s t, P\rangle,
$$

where $r w \in\{0,1\}$ denotes if particular employee is working remotely, st defines current state of the employee from possible states and $P$ defines a tuple of probabilities which define behaviour of particular agent. In the proposed model for the Employee following states are defined:

- Idle - agent is performing only job activities not related to the communication. 
- Reading text-related inbound message.

- Writing new text-related outbound message.

- Calling - performing direct call or onlinemeeting communication with another agent.

- Fault - a state, when infrastructure or security related fault has occurred, and it is not possible for agent to perform communication related work activity.

- Offline - employee is temporarily offline and is not accessible for direct communication.

Employee agents perform state transitions according to the following state chart (see Fig. 10).

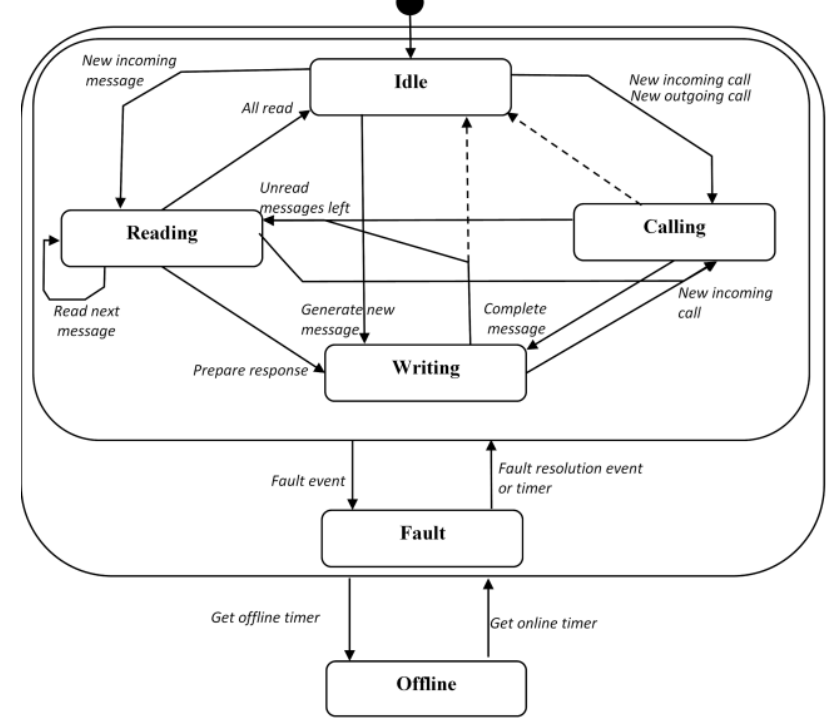

Fig. 10. State chart of Employee agent

Transition between states of the Employee agent could be induced: 1) by timer; 2) by model events; 3 ) by events from other Employee agents. Some of the transitions are occurring randomly with distinct, defined in the model parameters probability. E.g., Calling state could be caused with a certain probability from Idle state, if both random events occur: 1) to create a new communication, and 2) random selection of communication channel falls on call or online-meeting communication.

Offline and Fault states here are blocking communication activity of Employee agent for a certain random time period or until a particular fault resolution event occurs. In these states agent is not accessible by direct communication (e.g. onlinemeeting), but can receive and queue indirect messages (e.g. e-mail).

In its structure, the Employee agent contains inbox queues for incoming text messages. The agent will process these queues to reflect this message type related behaviour.

The Message agent can be used to define different types and context of the communication process between employees. Following message types are defined within the model: E-mail; Chat; Local information system messages; Phone Call; Online Meeting. Distinguishing different types of communication messages allows more robust modelling of communication processes within remote work context. Message object in terms of (6) is formalised as following structure:

$$
m e=\left\langle e m_{s}, e m_{r}, g, t_{b}, d\right\rangle,
$$

where $\mathrm{em}_{s}$ is message sender, êm is message receiver, $g$ is message type, $t_{b}$ time when message was sent, $d$ total proposed duration of message.

Remaining Infrastructure agent is proposed to model infrastructure specific behaviour, such as particular infrastructure component's faults on the communication flow in the system.

\subsubsection{Implementation}

To implement a model for Remote Workplace digital twin, the MESA agent-based simulation framework in Python [30] is applied, which allows development of agent-based models and includes built-in tools for organised collection of simulation output data and provides tools for visualisation of network-based environments. Moreover, the developed model is easier to integrate within a multi-modelling approach of resilient service infrastructure with other Python based components.

In order to simplify the implementation for faster initial experimental analysis, only a single agent class is used for the Employee agent. Other agents (such as Message) are not introduced as these should be created and destroyed too often but will have no interaction themselves. In turn Infrastructure behaviour is implemented only as employee state transitions by randomised timers and probabilities

To calculate key performance indicators of secure remote workplace in the digital twin a number of communication events is calculated each step as a number of all active connection sessions. This indicator is calculated each step as:

$$
N_{\text {comm }}(t)=|M E|,
$$

where $N_{\text {comm }}(t)$ is number of communications in time $t$, if set $M E$ has only active messages.

Time spent on the communication is processed as a relative indicator and is calculated for each agent as a number of steps where the agent was involved in communication divided over the whole model time. Duration of communication for each agent is processed as the actual duration of last 
communication activity. The last for all active messages in $M E$ is calculated as follows:

$$
D_{\text {comm }}(t)=\sum_{m e \in M E} t-t_{b}
$$

In (10), $D_{\text {comm }}$ denotes communication time key performance, $t$ denotes a current simulation time and $t_{b}$ is particular start time of each message.

\subsubsection{Experimental Analysis}

An experimental analysis of the remote workplace model is performed by application of digital twin with different combinations of model contextrelated and agent behaviour-related input parameters. Experimental results of a sample run are given in Fig. 11. Within this sample run the following model parameters were used: number of employees: 40; proportion of employees working remotely: $40 \%$; communication fault probability: 0.002 ; fault probability multiplier for remotely connected employees: 2.0 .

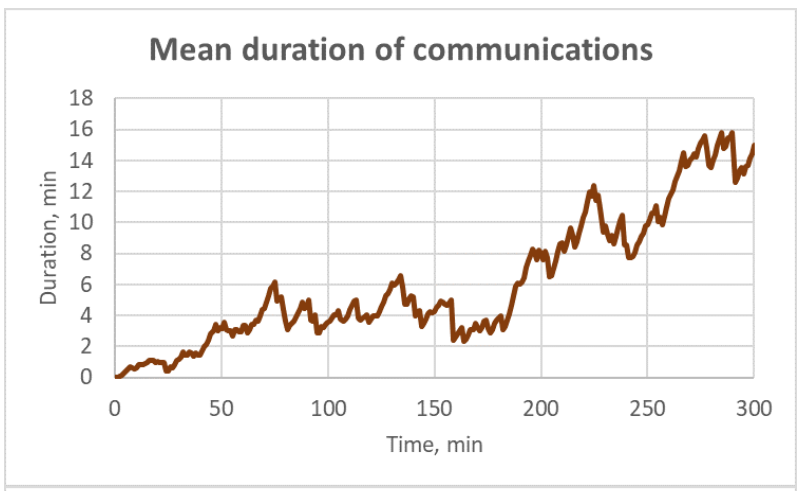

Proportion of time spent on communications

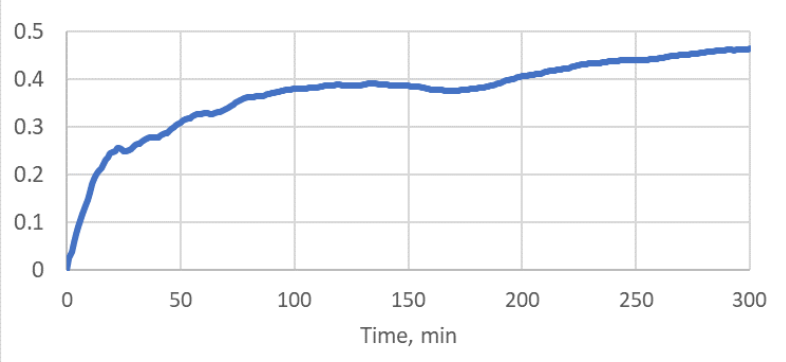

Fig. 11. Key performance indicators timelines in experiment with remote workplace digital twin

Experimental analysis shows that the developed model of the remote workplace digital twin should be very scrupulously tuned in parameters and internal configuration to comply with the actual communication network and communication behaviour of organisations employees.

In the future research the model should be extended to provide more accurate simulation. Also, additional factors affecting the system behaviour, such as more detailed security context or impact of organisation's structure and internal hierarchy on agent interaction, should be added.

\section{Conclusion}

The objective of the proposed multi-model approach was to provide a technological foundation for the analysis and improvement of resilient services. The proposed composite digital twin platform has the following benefits:

- Conduct simulations \& experiments: the approach provides a mechanism to do interactive simulations, where it is possible to experiment on the twin rather than on the real entity. Simulations are particularly useful when applying different modelling methods by using data from multiple sources.

- Share data across multiple data sources and storages: The developed technology provides tools to better understand the relationship between all the used data sources and storages in the context of the expected results therefore the data can be effectively shared across the different silos.

- Manage operations in real-time: the technology is able to support real-time operations by providing real-time visibility, getting recommendations, and creating actions based on events in the data therefore creating a situational awareness by building a holistic picture of the represented entities.

- Enable collaboration: The developed technology enables collaboration across the users in a multiuser web environment by providing a shared understanding of what an entity looks like when it is represented in a digital format.

The performed experiments show that the multimodel approach is a viable and promising solution for the development of digital twins for complex cyber-physical systems. A possible future direction for further research studies could be an integration of another existing M\&S methodologies, e.g., Markov Models [31] or Discrete Event System Specification (DEVS) [24].

\section{References:}

[1] A. Hughes, Forging the digital twin in discrete manufacturing: a vision for unity in the virtual and real worlds. LNS Research, 2018.

[2] M. Grieves, "Origins of the Digital Twin Concept," 2016.2 doi: 
10.13140/RG.2.2.26367.61609.

[3] J. Banks, J. II Carson, B. Nelson, and D. Nicol, Discrete-Event System Simulation, 5th ed. Pearson, 2010.

[4] J. D. Sterman, Business dynamics : systems thinking and modeling for a complex world. Boston: Irwin/McGraw-Hill, 2000.

[5] RTU, "ARTSS: Advanced Resilience Technologies for Secure Services," 2020. https://www.rtu.lv/en/university/rtuprojects/open?project_number $=4356$.

[6] Industrial Internet Consortium, "Digital Twins for Industrial Applications: Definition, Business Values, Design Aspects, Standards and Use Cases," 2020. [Online]. Available:

https://www.iiconsortium.org/pdf/IIC_Digita 1_Twins_Industrial_Apps_White_Paper_202 0-02-18.pdf.

[7] ISO, "ISO/DIS 23247-1. Automation systems and integration - Digital Twin framework for manufacturing," 2020.

[8] J. Koziorek, A. Gavlas, J. Konecny, M. Mikolajek, R. Kraut, and P. Walder, "Automated control system design with model-based commissioning," Int. J. Circuits, Syst. Signal Process., vol. 13, pp. 6-12, 2019.

[9] K. Sandkuhl and J. Stirna, "Supporting Early Phases of Digital Twin Development with Enterprise Modeling and Capability Management: Requirements from Two Industrial Cases BT - Enterprise, BusinessProcess and Information Systems Modeling," 2020, pp. 284-299.

[10] A. Bolton et al., "Gemini Principles," 2018. doi: https://doi.org/10.17863/CAM.32260.

[11] M. W. Grieves, "Virtually Intelligent Product Systems: Digital and Physical Twins," in Complex Systems Engineering: Theory and Practice, American Institute of Aeronautics and Astronautics, Inc., 2019, pp. 175-200.

[12] L. Wright and S. Davidson, "How to tell the difference between a model and a digital twin," Adv. Model. Simul. Eng. Sci., vol. 7, no. 1 , p. 13, Dec. 2020, doi: $10.1186 / \mathrm{s} 40323-$ 020-00147-4.

[13] Siemens, "Our Story: Digital Twin," 2020. https://www.plm.automation.siemens.com/gl obal/en/our-story/glossary/digital-

twin/24465 (accessed Dec. 13, 2020).

[14] STAMFORD, "Gartner Survey Reveals 47\% of Organizations Will Increase Investments in IoT Despite the Impact of COVID-19," 2020. https://www.gartner.com/en/newsroom/press -releases/2020-10-29-gartner-survey-reveals47-percent-of-organizations-will-increaseinvestments-in-iot-despite-the-impact-ofcovid-19- (accessed Dec. 13, 2020).

[15] AI.Multiple, "15 Digital Twin Applications/ Use Cases by Industry," 2020. https://research.aimultiple.com/digital-twinapplications (accessed Dec. 13, 2020).

[16] A. Litvinenko and A. Aboltins, "Selection and performance analysis of chaotic spreading sequences for DS-CDMA systems," in 2016 Advances in Wireless and Optical Communications (RTUWO), 2016, pp. 38-45, doi: 10.1109/RTUWO.2016.7821852.

[17] P. Viskup and K. Vichova, "The impact of crisis situations to the transport service of the territory for the selected hospital," Int. J. Circuits, Syst. Signal Process., vol. 13, pp. 73-78, 2019.

[18] V. Kulkarni, S. Barat, and T. Clark, "Towards Adaptive Enterprises Using Digital Twins," in 2019 Winter Simulation Conference (WSC), 2019, pp. 60-74, doi: 10.1109/WSC40007.2019.9004956.

[19] S. Barat, V. Kulkarni, T. Clark, and B. Barn, "An Actor Based Simulation Driven Digital Twin For Analyzing Complex Business Systems," in 2019 Winter Simulation Conference (WSC), Dec. 2019, vol. 2019Decem, pp. 157-168, doi: 10.1109/WSC40007.2019.9004694.

[20] M. Liu, S. Fang, H. Dong, and C. Xu, "Review of digital twin about concepts, technologies, and industrial applications," $J$. Manuf. Syst., 2020, doi: 10.1016/j.jmsy.2020.06.017.

[21] Q. Qi et al., "Enabling technologies and tools for digital twin," J. Manuf. Syst., 2019, doi:

https://doi.org/10.1016/j.jmsy.2019.10.001.

[22] B. Schoenberg, M. Adams, and T. Fiddaman, "How to create web simulations with Vensim using Forio Simulate | Vensim," 2011. https://vensim.com/video-foriosimulate/ (accessed Dec. 12, 2020).

[23] K. Sandkuhl and J. Stirna, Capability Management in Digital Enterprises. 2018.

[24] B. Zeigler, A. Muzy, and E. Kofman, Theory of Modeling and Simulation: Discrete Event \& Iterative System Computational Foundations, 3rd Editio. Academic Press, 2018.

[25] C. Cronrath, L. Ekström, and B. Lennartson, 
"Formal Properties of the Digital Twin Implications for Learning, optimization, and Control," in 2020 IEEE 16th International Conference on Automation Science and Engineering (CASE), 2020, pp. 679-684, doi: 10.1109/CASE48305.2020.9216822.

[26] Likumi.lv, "Republic of Latvia Cabinet Regulation No. 555 (Adopted 28 August 2018): Procedures for the Organisation of and Payment for Health Care Services," 2018. https://likumi.lv/ta/en/en/id/301399 (accessed Nov. 24, 2020).

[27] dati.gov.lv, "The Centre for Disease Prevention and Control of Latvia: COVID$19, "$ 2020.

https://ata.gov.lv/dati/lv/dataset/covid19/resource/d499d2f0-b1ea-4ba2-96002c701b03bd4a?inner_span=True (accessed Dec. 10, 2020).

[28] CSB, "Central Statistical Bureau of Latvia: Basic indicators of health care services at the end of the year 2019." https://data.csb.gov.lv/pxweb/en/sociala/soci ala_veseliba_iestades/VAG010.px/table/ta bleViewLayout1 (accessed Dec. 13, 2020).

[29] W. M. P. van der Aalst, Process Mining: Discovery, Conformance and Enhancement of Business Processes. Berlin, Heidelberg: Springer-Verlag, 2011.

[30] D. Masad and J. Kazil, "Mesa: An AgentBased Modeling Framework," in Python in Science Conference, 2015, pp. 51-58, doi: 10.25080/Majora-7b98e3ed-009.

[31] E. Chromy and B. Ivan, "Complex mathematical model of the contact center with determining of the optimal number of agents," Int. J. Circuits, Syst. Signal Process., vol. 12, pp. 488-493, Jan. 2018.

\section{Contribution of individual authors to the creation of a scientific article}

Arnis Lektauers has contributed to the design and prototyping of the multi-model approach for simulation-based digital twin framework.

Jel̦ena Pečerska has contributed to the review of existing digital twin technologies, has implemented the simulation model of secure telemedicine services.

Vitâlijs Boḷšakovs has contributed to the review of existing digital twin technologies, has implemented the digital twin of secure workplace.

Andrejs Romānovs has contributed to analysis of the state of the art in digital twins, as well as the design of the digital twin concept for the use case of telemedicine services.

Jānis Grabis has contributed to the high-level ideology and design of the extended CDD method used as a foundation for the digital twin framework. Artis Teilāns has contributed to the high-level ideology and design of the multi-model approach for simulation-based digital twin framework.

\section{Sources of funding for research presented in a scientific article or scientific article itself}

This research is funded by the Ministry of Education and Science, Republic of Latvia, project ARTSS, project No. VPP-COVID-2020/1-0009.

\section{Creative Commons Attribution License 4.0 (Attribution 4.0 International , CC BY 4.0)}

This article is published under the terms of the Creative Commons Attribution License 4.0 https://creativecommons.org/licenses/by/4.0/deed.en US 